\title{
DESCRIÇÃO FITOSSOCIOLÓGICA DA ARBORIZAÇÃO DE RUAS POR MEIO DE DIFERENTES FORMAS DE EXPRESSÃO DA DOMINÂNCIA E DA DENSIDADE
}

\author{
PHYTOSOCIOLOGICAL DESCRIPTION OF STREET TREES BY DIFFERENT WAYS OF \\ DOMINANCE AND DENSITY EXPRESSION
}

\author{
Rogério Bobrowski ${ }^{1}$ Rinaldo Luiz Caraciolo Ferreira ${ }^{2}$ Daniela Biondi ${ }^{3}$
}

\begin{abstract}
RESUMO
As ferramentas que possibilitam a análise da arborização de ruas incluem as descrições fitossociológicas para expressão da importância das espécies. Para este trabalho foram coletadas informações de 15 unidades amostrais de um inventário realizado em 2010 na cidade de Curitiba - PR. Com base nisso foram calculados os índices descritores da fitossociologia, com dominância expressa pelo DAP (m) e pela Área de Copa $\left(\mathrm{m}^{2}\right)$ para a obtenção de distintos Valores de Importância (VI), e com densidade expressa pelo índice de performance da espécie (IPE) e dominância expressa pela Área de Copa $\left(\mathrm{m}^{2}\right)$ para o cálculo de outro VI. Os resultados demonstraram que a classificação das espécies pela frequência relativa (proporção de indivíduos) exalta a importância das espécies com maior número de árvores e que a classificação das espécies pelo VI obtido pela dominância descrita pelo DAP tende a exaltar a importância daquelas com maior tamanho de tronco. Por outro lado, a obtenção do VI pela dominância descrita pela área de copa tende a expressar melhor a participação das espécies na composição da arborização de ruas e a obtenção do VI por meio do índice de performance da espécie demonstra mais claramente a abundância, as dimensões e a condição das árvores.
\end{abstract}

Palavras-chave: comunidade arbórea; silvicultura urbana; ecologia urbana; floresta urbana.

\begin{abstract}
Tools that make possible street tree analysis include phytosociological descriptions to express relative species importance. To this work, it was collected information from 15 unit plots of an inventory held in 2010 in the city of Curitiba, in Paraná state. On this base, the phytosociological description indexes were calculated with dominance expressed by DBH $(\mathrm{m})$ and by Crown Area $\left(\mathrm{m}^{2}\right)$ to obtain distinct Importance Values (VI), and with density expressed by species performance index (SPI) and dominance expressed by Crown Area $\left(\mathrm{m}^{2}\right)$ to calculate another VI. Results showed that species classification by relative frequency (proportion of individuals) exalt the importance of species with a greater number of trees and that species classification by VI obtained by dominance described by DBH tend to exalt the importance of those with a greater trunk size. On the other hand, the attainment of VI by dominance described by crown area tend to express a better species participation on street tree composition and the attainment of VI by the species performance index shows more clearly the abundance, the dimensions and the condition of the trees.
\end{abstract}

Keywords: tree community; urban forestry; urban ecology; urban forest.

1 Engenheiro Florestal, Dr., Professor Ajunto do Departamento de Engenharia Florestal, Universidade Estadual do Centro-Oeste, BR 153, Km 7, Riozinnho, CEP 84500-000, Irati (PR), Brasil. rogerio@irati.unicentro.br

2 Engenheiro Florestal, Dr., Professor Associado do Departamento de Ciências Florestais, Universidade Federal Rural de Pernambuco, Rua Dom Manoel de Medeiros, s/n, Bairro Dois Irmãos, CEP 52171-900, Recife (PE), Brasil. Bolsista Produtividade em Pesquisa do CNPq. rinaldof@ufrpe.br

3 Engenheira Florestal, Dr ${ }^{\mathrm{a}}$., Professora Associada do Departamento de Ciências Florestais, Universidade Federal do Paraná, Av. Pref. Lothário Meissner, 632, Jardim Botânico, Campus III, CEP 80210-170, Curitiba (PR), Brasil. Bolsista Produtividade em Pesquisa do CNPq.dbiondi@ufpr.br

Recebido para publicação em 27/02/2014 e aceito em 4/03/2015

Ci. Fl., v. 26, n. 4, out.-dez., 2016 


\section{INTRODUÇÃO}

A obtenção de dados por meio de inventários florestais e posterior análise são procedimentos básicos para o planejamento e gestão da arborização de ruas, bem como para a gestão de áreas verdes, ambas componentes da arborização urbana de uma cidade (BOBROWSKI, 2011b).

Atualmente, os inventários florestais da arborização urbana realizados no Brasil tendem a apresentar a ordenação das espécies utilizando apenas a frequência ou a proporção de cada uma delas como fator descritivo, não fazendo uso da totalidade dos componentes da análise fitossociológica (frequência, densidade, dominância e valor de importância). Tais características de análise incompleta podem ser observadas nos trabalhos de Sampaio e De Angelis (2008), Rossatto et al. (2008), Silva et al. (2008), Thaiutsa et al. (2008), Moura e Santos (2009), Kuruneri-Chitepo e Shackleton (2011), Lima e Silva Junior (2011), Souza (2011), Sreetheran et al. (2011), Kramer e Krupek (2012), dentre outros. Exceções a isso e que adotam a abordagem fitossociológica completa podem ser observadas no trabalho de Romani (2011), Nagendra e Gopal (2010) e Muthulingam e Thangavel (2011).

Romani (2011) fez uso das ferramentas de análise fitossociológica para estudar a composição e a estrutura da vegetação arbórea componente de uma praça na cidade de Ribeirão Preto, São Paulo. Para isso, utilizou a densidade, a dominância e o valor de cobertura para a análise da estrutura horizontal, a fim de compreender a importância relativa das espécies vegetais avaliadas nesta área verde. Da mesma forma, Muthulingam e Thangavel (2011) aplicaram a ferramenta de análise fitossociológica para estudo da composição e estrutura da vegetação avaliada em diferentes áreas verdes do Distrito de Chennai, na Índia.

$\mathrm{Na}$ arborização de ruas, a análise da composição e estrutura por meio das ferramentas da fitossociologia, se aplicada, não é divulgada no Brasil, porém, para inventários realizados em cidades dos Estados Unidos da América observase isso como uma prática necessária, tal como nos trabalhos de Maco (2003), McPherson e Simpson (2002), McPherson et al. (2005), Galvin (2007), Nowak et al. (2008), Davey Resource Group (2010), Davey Resource Group (2012), DiSalvo et al. (2012). A diferença metodológica reside na descrição dos componentes da análise fitossociológica, pois a área de cobertura por copas e a área foliar da copa (densidade de copa) assumem papel mais importante que a área basal na descrição da dominância das espécies na arborização de ruas. A área de copa das árvores sob crescimento livre, sem competições, pode expressar mais adequadamente o crescimento e as características da espécie (HASENAUER, 1997), o que tende a condicionar melhor expansão da copa. Para Bobrowski e Biondi (2012), a copa na arborização de ruas é o principal ponto de destaque das árvores, sendo por meio dela que se sucedem os benefícios ambientais e estéticos como redução da amplitude térmica, absorção e filtragem da água da chuva e a beleza estética da floração.

As informações quantitativas obtidas e ordenadas por meio da análise fitossociológica possibilita caracterizar e comparar composições florísticas da arborização de ruas de bairros ou cidades diferentes e/ou realizar a comparação entre períodos de avaliação distintos, a fim de verificar mudanças estruturais mensuráveis, ao modo do que se faz nos estudos fitossociológicos de florestas nativas, tal como nos trabalhos de Martins et al. (2006), Araujo et al. (2010), Costa et al. (2011) e Silva et al. (2012).

Semenzato et al. (2011), em trabalho realizado na arborização urbana de quatro cidades italianas (Piazzola sul Brenta, Pontelongo, Vigonovo e Piove di Sacco), utilizaram a área de copa como fator descritivo da dominância no cálculo do VI. Os autores procederam desta forma tendo em vista a dificuldade em se utilizar o cálculo do VI à maneira de trabalhos como os de McPherson et al. (2005) e Nowak et al. (2008), os quais utilizam a abundância relativa, a área de copa relativa e a área foliar relativa (densidade de copa) para a determinação do valor de importância de árvores urbanas.

A partir deste contexto e considerando a importância de se conhecer a estrutura da composição de espécies na arborização de ruas de Curitiba, os objetivos do presente trabalho foram avaliar e analisar diferentes métodos de classificação das espécies pela obtenção do Valor de Importância (VI) a partir do DAP (m), da Área de Copa $\left(\mathrm{m}^{2}\right)$ e do Índice de Performance da Espécie (IPE), comparando as mudanças observadas no posicionamento das espécies entre estas formas de obtenção do VI e em relação ao meio tradicionalmente utilizado com a descrição da proporção do número de indivíduos amostrados. 


\section{MATERIAL DE MÉTODOS}

Os dados utilizados nesta pesquisa são provenientes de um inventário da arborização de ruas realizado na cidade de Curitiba, Paraná, no ano de 2010 por Bobrowski (2011a). Esta cidade está localizada na região leste do Estado, no primeiro planalto paranaense, entre as coordenadas $25^{\circ} 25^{\prime} 48^{\prime \prime}$ 'S e 4916'5”O (BOBROWSKI, 2011a).

A preocupação com a arborização de ruas da cidade remonta ao século XIX, com a primeira ordem pública para custeio da arborização. Entretanto, somente em 1967 é que houve a introdução de espécies nativas como Handroanthus chrysotrichus (ipê-amarelo-miúdo), Handroanthus albus (ipê-amarelo), Handroanthus heptaphyllus (ipê-roxo), Tibouchina sellowiana (quaresmeira) e Poincianella pluviosa (sibipiruna), sendo que até esse ano a composição era feita com espécies exóticas como Acer negundo (acer), Ligustrum lucidum (alfeneiro), Melia azedarach (cinamomo) e Jacaranda mimosaefolia (jacarandá) (BIONDI; ALTHAUS, 2005). Dados de Bobrowski, Filho (2012) e Bobrowski e Biondi (2012) apontam que a arborização da cidade apresenta características de estágio de amadurecimento, devido ao aumento do número de árvores nas maiores classes de DAP e Área de Copa entre 1984 e 2010, e indícios de sustentabilidade, devido à presença de árvores em todas as classes de DAP e à distribuição unimodal dos dados. Portanto, na arborização de ruas de Curitiba encontram-se árvores jovens, adultas e senescentes, em diferentes condições estruturais e fitossanitárias (BOBROWSKI, 2011a).

Os dados avaliados e analisados foram coletados em 15 unidades amostrais de reamostragem de um inventário da arborização de ruas, com dimensões de 500 X $500 \mathrm{~m}$ cada uma. As informações obtidas em cada parcela foram: espécie; altura total $(\mathrm{m})$, obtida por meio de um hipsômetro de Blume-Leiss; perímetro a altura do peito $(\mathrm{cm})$, obtido por meio de fita métrica e posteriormente convertido a diâmetro a altura do peito $(\mathrm{m})$; diâmetro de copa (m), obtido por estimativa a partir de quatro raios de medição $(\mathrm{d}$ - raio para direita, $\mathrm{e}$ - raio para esquerda, $\mathrm{r}$ - raio para rua, $\mathrm{c}$ - raio para construção) tomados com auxílio de uma trena; altura da fiação (m), correspondente à rede de telecomunicações, obtida por meio de um hipsômetro de Blume-Leiss; altura de bifurcação (m), obtida por meio de uma trena; área do canteiro no qual estava a árvore $\left(\mathrm{m}^{2}\right)$, posicionamento da árvore (distância da árvore ao meio fio (m), distância da árvore ao muro ou construção $(\mathrm{m})$, distância da árvore à projeção da rede de energia/telefone $(\mathrm{m})$ e espaçamento de plantio (m)) obtidos com o auxílio de uma trena. A avaliação da condição geral das árvores foi realizada conforme descrito por Bobrowski (2011a).

A análise da estrutura horizontal de composição da arborização de ruas foi feita por meio de índices de descrição fitossociológica, de acordo com as orientações de Moro e Martins (2011). De posse dos dados do inventário analisou-se a composição da estrutura horizontal por meio dos fatores: frequência (absoluta e relativa), densidade (absoluta e relativa), dominância (absoluta e relativa) e valor de importância (VI). Da mesma forma foi utilizada a proporção da espécie (número total de indivíduos da espécie em relação ao número total de indivíduos amostrados) para a descrição da importância no inventário analisado (Método S1).

Para a determinação do valor de importância foram utilizados três métodos: S2) VI descrito a partir da dominância determinada por meio do DAP (m); S3) VI descrito a partir da dominância determinada por meio da área de copa $\left(\mathrm{m}^{2}\right)$; S4) VI descrito a partir da dominância determinada pela área de copa e a densidade substituída pelo índice de performance da espécie (IPE).

Esta última forma de determinação do VI foi adotada como metodologia para compreender a influência da condição geral das árvores e das espécies na determinação do valor de importância e no posicionamento das espécies com relação a este índice.

O Índice de Performance da Espécie (IPE) utilizado foi adaptado dos trabalhos de McPherson et al. (2003), Peper et al. (2004), Freilicher (2010) e Davey Resource Group (2010) e serve para expressar a proporção de árvores, de cada espécie, que estão em condições boas e satisfatórias tomando por base a condição total das árvores amostradas (boas, satisfatórias, ruins e mortas), sendo: árvore boa, vigorosa: aquela apresentando arquitetura típica da espécie, sem sinais de pragas, doenças ou injúrias; árvore satisfatória: aquela com vigor médio, pequenos problemas com pragas, doenças ou danos físicos; árvore ruim: aquela em estado geral de declínio, severos danos de pragas, doenças ou danos físicos; árvore morta ou com morte iminente.

O Índice de Performance da Espécie foi obtido pela equação: 


$$
I P E=\frac{\left(\frac{n a B+n a S}{n}\right) * 100}{\left(\left(\frac{N A B+N A S}{N}\right) * 100\right)}
$$

Em que: naB = número de árvores da espécie "e" que estão na classe de condição Boa; naS = número de árvores da espécie "e" que estão na classe de condição Satisfatória; $\mathrm{n}=$ número de árvores da espécie "e"; $\mathrm{NAB}=$ número total de árvores amostradas na condição Boa; NAS = número total de árvores amostradas na condição Satisfatória; $\mathrm{N}=$ número total de árvores amostradas no trabalho.

A partir dos valores relativos dos resultados obtidos, tanto da forma usual de descrição das espécies nos inventários da arborização de ruas (Método S1) quanto dos métodos de determinação do valor de importância (Métodos S2, S3 e S4), foi realizada análise por meio do Teste de Kruskal-Wallis (SIEGEL; CASTELLAN, 2006), a fim de verificar diferenças entre os métodos de determinação do VI e consequentes postos assumidos pelas espécies (rankings). Esta análise foi realizada via software $\mathrm{R}$, por meio do pacote "Stats" (R CORE TEAM, 2014). Em seguida foi aplicado um teste post hoc, conforme sugerido por Siegel e Castellan (2006), para identificação de diferenças significativas entre as médias dos ranks dos pareamentos possíveis entre os métodos. Este teste foi realizado por meio do pacote "PMCMR" (POHLERT, 2014).

Os dados da análise comparativa entre os métodos de obtenção da importância das espécies também foram submetidos ao Teste de correlação por postos de Kendall (SIEGEL; CASTELLAN, 2006) para obtenção do coeficiente de correlação entre os postos assumidos pelas espécies. Este teste foi realizado via pacote "Kendall" (MCLEOD, 2011) do software R.

Da mesma forma, todos os valores relativos de importância obtidos nos métodos S1, S2, S3 e S4 foram submetidos à análise de correlação com os valores relativos de IPE, via pacote "Stats" ( $R$ CORE TEAM, 2014) para verificar a existência ou ausência de influência do padrão de condição das árvores na determinação da importância das espécies.

\section{RESULTADOS E DISCUSSÃO}

Ao todo foram analisadas 4360 árvores distribuídas em 122 espécies diferentes nas quinze unidades amostrais avaliadas. Os dados da análise fitossociológica para as 20 principais espécies estão apresentados nas Tabelas 1, 2 e 3 e Figura 1 e correspondem a $50 \%$ do valor de importância obtido em cada método.

Por meio do Teste de Kruskal-Wallis constatou-se diferença significativa entre os métodos de obtenção do valor de importância das espécies $\left(\chi_{\mathrm{KW}}^{2} 75,48 ; \mathrm{GL}=121, p\right.$-valor $\left.=2,85^{-16}\right)$, sendo que apenas os métodos S2 e S3 não diferiram significativamente entre si $(p$-valor $=0,99)$ quando analisados pelo teste post hoc. Isto evidencia que o método tradicional de descrição da importância das espécies (S1) posiciona-as de forma significativamente diferente em relação aos demais métodos (S2, S3 e S4). Por conta disso, os trabalhos de inventário da arborização de ruas (amostral ou censo) que representam a importância das espécies por meio da proporção de indivíduos amostrados podem não expressar adequadamente a composição da estrutura da arborização de ruas e tampouco a importância das espécies.

Apesar da proporção de indivíduos ser utilizada como um indicativo da adequabilidade da diversidade de espécies na composição da arborização de ruas (SAMPAIO; DE ANGELIS, 2008; SILVA et al., 2008; SARTORI; BALDERI, 2011; SOUSA tal., 2013; SCHALLEN-BERGER; MACHADO, 2014), pautando recomendações de planejamento e manejo, Richards (1993) e Raupp et al. (2006) afirmam que a adaptabilidade das espécies às condições de estresse urbano é que deve ser o objetivo primordial de planejamento. Desta forma, o uso da proporção de indivíduos como descrição da importância das espécies pode desprestigiar os benefícios que possam ser oferecidos pelas árvores, mesmo de espécies que estejam em proporção acima de valores hipotéticos recomendados. Desta forma, decisões de gestão do patrimônio arbóreo nas calçadas podem não ser apropriadas, se apoiadas por esta técnica.

Soares et al. (2011) ao analisarem a arborização de ruas da cidade de Lisboa, Portugal, afirmaram que a elevada proporção das espécies Celtis ocidentalis, Tilia sp. e Jacaranda mimosifolia era um fator preocupante por conta de pragas e fatores estressantes que podem dizimá-las, entretanto, não consideraram em suas análises a condição geral dos indivíduos amostrados e tampouco a sua representatividade em termos de área de copa, já que esta é a principal via de oferta de benefícios ambientais e estéticos (BOBROWSKI; BIONDI, 2012). Esta preocupação com a proporção dos indivíduos e a forma de análise parcial da importância 
TABELA 1: Número de indivíduos amostrados $(\mathrm{N})$ e valores relativos para a frequência (FR), densidade (DR), dominância (DoR) e valor de importância (VI) dos métodos S1(Mét S1) e S2 (Mét S2) de análise fitossociológica para a arborização de ruas de Curitiba - PR.

TABLE 1: Number of individuals sampled (N) and relative values for the frequency (FR), density (DR), dominance (DoR) and importance value (VI) of methods S1 (Mét S1) and S2 (Mét S2) of phytossociology analysis for street trees of Curitiba, PR state.

\begin{tabular}{lcccccc}
\hline & \multirow{2}{*}{ Espécies } & Nét S1 & \multicolumn{5}{c}{ Mét S2 } \\
\cline { 5 - 8 } & & Prop & FR & DR & DoR & VI \\
\hline Ligustrum lucidum W.T. Aiton & 531 & 12,18 & 2,77 & 12,18 & 16,63 & 10,52 \\
Tipuana tipu (Benth.) Kuntze & 277 & 6,35 & 2,13 & 6,35 & 20,06 & 9,51 \\
Lagerstroemia indica L. & 705 & 16,17 & 3,19 & 16,17 & 4,04 & 7,80 \\
Parapiptadenia rigida (Benth.) Brenan & 194 & 4,45 & 1,28 & 4,45 & 13,59 & 6,44 \\
Handroanthus chrysotrichus (Mart. ex A.DC.) Mattos & 423 & 9,70 & 2,55 & 9,70 & 2,38 & 4,88 \\
Handroanthus albus (Cham.) Mattos & 272 & 6,24 & 2,98 & 6,24 & 4,34 & 4,52 \\
Acer negundo L. & 204 & 4,68 & 2,13 & 4,68 & 4,51 & 3,77 \\
Lafoensia pacari A.St.-Hil. & 236 & 5,41 & 2,77 & 5,41 & 2,08 & 3,42 \\
Anadenanthera colubrina (Vell.) Brenan & 60 & 1,38 & 1,91 & 1,38 & 5,81 & 3,03 \\
Melia azedarach L. & 87 & 2,00 & 2,77 & 2,00 & 3,00 & 2,59 \\
Cassia leptophylla Vogel & 120 & 2,75 & 1,49 & 2,75 & 3,43 & 2,56 \\
Handroanthus heptaphyllus (Martius) Mattos & 119 & 2,73 & 2,13 & 2,73 & 1,61 & 2,16 \\
Syagrus romanzoffiana (Cham.) Glassman & 72 & 1,65 & 2,55 & 1,65 & 2,02 & 2,07 \\
Poincianella pluviosa var. peltophoroides (Benth.) L.P.Queiroz & 130 & 2,98 & 2,13 & 2,98 & 0,82 & 1,98 \\
Jacaranda mimosifolia D. Don & 49 & 1,12 & 2,13 & 1,12 & 2,20 & 1,82 \\
Senna macranthera (DC. ex Collad.) H.S. Irwin \& Barneby & 63 & 1,44 & 2,98 & 1,44 & 0,55 & 1,66 \\
Hibiscus rosa-sinensis L. & 87 & 2,00 & 2,55 & 2,00 & 0,36 & 1,64 \\
Libidibia ferrea var. leiostachya (Benth.) L.P.Queiroz & 69 & 1,58 & 1,28 & 1,58 & 1,06 & 1,31 \\
Eugenia uniflora L. & 47 & 1,08 & 2,55 & 1,08 & 0,13 & 1,25 \\
Araucaria angustifolia (Bertol.) Kuntze & 28 & 0,64 & 1,70 & 0,64 & 1,34 & 1,23 \\
\hline
\end{tabular}

das espécies em um inventário da arborização de ruas também é comum em trabalhos realizados em cidades brasileiras (SAMPAIO; DE ANGELIS, 2008; ROSSATTO et al., 2008; MOURA; SANTOS, 2009; SARTORI; BALDERI, 2011; SCHALLENBERGER; MACHADO, 2014).

A partir da análise comparativa dos VIs obtidos pelos métodos S2, S3 e S4 em relação ao método S1constatou-se que houve mudança para um posicionamento melhor em 45,90\%, 50,00\% e $45,08 \%$, respectivamente (Tabela 3 ).

Por exemplo, a espécie mais frequente no inventário realizado (Lagerstroemia indica 705 indivíduos) assume a maior importância na composição florística se a mesma é analisada por meio da expressão da proporção de indivíduos em relação ao total amostrado. Entretanto, se a composição da arborização é analisada por meio do VI com a dominância descrita pela área basal, esta espécie perde importância (705 indivíduos, área basal $0,84 \mathrm{~m}^{2} / \mathrm{ha}$ ) ficando em terceira colocação, em detrimento a Ligustrum lucidum (531 indivíduos, área basal $3,47 \mathrm{~m}^{2} / \mathrm{ha}$ ), na primeira posição, e a Tipuana tipu (277 indivíduos, área basal $4,18 \mathrm{~m}^{2} /$ ha), na segunda posição. Da mesma forma, se considerado o VI com a dominância descrita pela área de copa a espécie Tipuana tipu (277 indivíduos, área de copa $2887,55 \mathrm{~m}^{2} / \mathrm{ha}$ ) é que assume maior importância na composição florística em relação as outras duas. Se considerada a composição do VI com informação da performance das espécies (IPE), as espécies Tipuana tipu (tipuana) e Parapiptadenia rigida (angico) assumem os melhores postos, havendo redução da importância das espécies 
TABELA 2: Valores relativos para a densidade (DR), dominância (DoR), índice de performance da espécie (IPE) e valor de importância (VI) dos Métodos S3 (Mét S3) e S4 (Mét S4) de análise fitossociológica para a arborização de ruas de Curitiba - PR.

TABLE 2: Relative values for density (DR), dominance (DoR), species performance index (IPE) and importance value (VI) of methods S3 (Mét S3) and S4 (Mét S4) of phytossociology analysis for street trees of Curitiba, PR state.

\begin{tabular}{lcccccc}
\hline \multirow{2}{*}{ Espécies } & \multicolumn{3}{c}{ Mét S3 } & \multicolumn{3}{c}{ Mét S4 } \\
\cline { 2 - 7 } & DR & DoR & VI & IPE & DoR & VI \\
\hline Ligustrum lucidum W.T. Aiton & 12,18 & 10,76 & 8,57 & 0,40 & 10,76 & 4,64 \\
Tipuana tipu (Benth.) Kuntze & 6,35 & 23,62 & 10,70 & 0,98 & 23,62 & 8,91 \\
Lagerstroemia indica L. & 16,17 & 5,17 & 8,18 & 0,87 & 5,17 & 3,08 \\
Parapiptadenia rigida (Benth.) Brenan & 4,45 & 13,17 & 6,30 & 1,12 & 13,17 & 5,19 \\
Handroanthus chrysotrichus (Mart. ex A.DC.) Mattos & 9,70 & 3,96 & 5,40 & 1,07 & 3,96 & 2,53 \\
Handroanthus albus (Cham.) Mattos & 6,24 & 4,57 & 4,60 & 1,08 & 4,57 & 2,88 \\
Acer negundo L. & 4,68 & 3,99 & 3,60 & 0,38 & 3,99 & 2,16 \\
Lafoensia pacari A.St.-Hil. & 5,41 & 1,79 & 3,32 & 0,97 & 1,79 & 1,84 \\
Anadenanthera colubrina (Vell.) Brenan & 1,38 & 5,43 & 2,91 & 0,96 & 5,43 & 2,77 \\
Melia azedarach L. & 2,00 & 5,43 & 2,40 & 0,57 & 5,43 & 1,93 \\
Cassia leptophylla Vogel & 2,75 & 4,03 & 2,76 & 0,97 & 4,03 & 2,17 \\
Handroanthus heptaphyllus (Martius) Mattos & 2,73 & 2,14 & 2,33 & 1,09 & 2,14 & 1,79 \\
Syagrus romanzoffiana (Cham.) Glassman & 1,65 & 1,16 & 1,79 & 1,09 & 1,16 & 1,60 \\
Poincianella pluviosa var. peltophoroides (Benth.) L.P.Queiroz & 2,98 & 1,35 & 2,15 & 0,80 & 1,35 & 1,42 \\
Jacaranda mimosifolia D. Don & 1,12 & 2,18 & 1,81 & 0,91 & 2,18 & 1,74 \\
Senna macranthera (DC. ex Collad.) H.S. Irwin \& Barneby & 1,44 & 0,64 & 1,69 & 0,57 & 0,64 & 1,40 \\
Hibiscus rosa-sinensis L. & 2,00 & 0,45 & 1,67 & 0,84 & 0,45 & 1,28 \\
Libidibia ferrea var. leiostachya (Benth.) L.P.Queiroz & 1,58 & 2,06 & 1,64 & 1,15 & 2,06 & 1,49 \\
Eugenia uniflora L. & 1,08 & 0,39 & 1,34 & 0,72 & 0,39 & 1,22 \\
Araucaria angustifolia (Bertol.) Kuntze & 0,64 & 0,94 & 1,09 & 1,12 & 0,94 & 1,25 \\
\hline
\end{tabular}

Ligustrum lucidum (alfeneiro), Lagerstroemia indica (extremosa) e de outras com condição geral pior do que as espécies com melhor desempenho (qualidade de estabelecimento e desenvolvimento).

O menor valor do índice de correlação por postos de Kendall $(\tau)$ observado entre S1 e S4 $(0,35)$ justifica a grande proporção de mudança da classificação das espécies, tanto para postos indicativos de menor importância na análise fitossociológica da arborização de ruas quanto para postos indicativos de maior importância, porém, com maior destaque para a situação "piorou posição".

Já em relação à classificação das espécies em ordem de importância, a partir do cálculo do VI com a dominância expressa pela área basal (S2), houve mudança para um melhor posicionamento em
$54,09 \%$ e $45,09 \%$ delas, respectivamente, para as situações S3 e S4. Entre as situações S3 e S4, houve melhoria no posicionamento das espécies no cálculo do VI para $45,08 \%$ delas. Para todas as situações comparativas, as menores proporções de mudanças ocorreram para o tipo "piorou mudança", em relação aos demais tipos de classificação constantes na Tabela 3. Da mesma forma, os menores valores de correlação por postos de Kendall foram observados para as situações em que houve maior proporção de mudanças para postos de menor importância.

As maiores proporções de mudança para o tipo de classificação "melhorou posição" foram observadas para as análises comparativas entre as situações S1-S3 e S2-S3. Nestas duas comparações, a área de copa utilizada como informação descritiva do fator dominância do VI é que promoveu as 
TABELA 3: Número de mudanças de posição entre diferentes formas de obtenção do valor de importância (VI), métodos S1, S2, S3 e S4, com respectivos valores do correlação por postos de Kendall $(\tau)$.

TABLE 3: Number of position changes among different ways of obtaining the importance value (IV), methods S1, S2, S3 e S4, with respective values of correlation by ranks of Kendall $(\tau)$.

\begin{tabular}{cccccc}
\hline \multirow{2}{*}{ Comparação } & $\begin{array}{c}\text { Melhorou } \\
\text { Posição }\end{array}$ & $\begin{array}{c}\text { Manteve } \\
\text { Posição }\end{array}$ & $\begin{array}{c}\text { Piorou } \\
\text { Posição }\end{array}$ & \multicolumn{2}{c}{ Teste de Kendall } \\
\cline { 5 - 6 } & 56 & 8 & 58 & 0,79 & $\left(2,22^{-16}\right)$ \\
S1-S2 & 61 & 7 & 54 & 0,83 & $\left(2,22^{-16}\right)$ \\
S1-S3 & 55 & 7 & 60 & 0,35 & $\left(7,87^{-9}\right)$ \\
S1-S4 & 66 & 26 & 30 & 0,89 & $\left(2,22^{-16}\right)$ \\
S2-S3 & 56 & 6 & 60 & 0,39 & $\left(1,91^{-10}\right)$ \\
S2-S4 & 55 & 7 & 60 & 0,44 & $\left(2,22^{-16}\right)$ \\
S3-S4 & & & & & \\
\hline
\end{tabular}

mudanças de posicionamento, com destaque das espécies que apresentaram maior área de copa em relação as outras (Tipuana tipu, Parapiptadenia rigida, Anadenanthera colubrina, Jacaranda mimosifolia, dentre outras), mesmo em relação a algumas espécies que apresentaram maior proporção de indivíduos na amostragem realizada. Isto é uma condição interessante na análise de composição florística da arborização de ruas, pois a copa é o principal meio de expressão dos benefícios almejados com as árvores nas calçadas, sejam eles ambientais ou estéticos (BOBROWSKI; BIONDI, 2012), principalmente para ruas ou avenidas que apresentam incômodo ambiental pela elevada amplitude térmica ou pela sensação de desconforto (MARTINI et al., 2013; MARTINI et al., 2013). Destaca-se aqui a maior correlação por postos $(\tau=$ 0,89 ) entre os métodos S2 e S3, para os quais não foi constatada diferença significativa por meio do teste post hoc $(p$-valor $=0,99)$, após análise pelo Teste de Kruskal-Wallis.

A utilização do Índice de Performance da Espécie (IPE) como um dos fatores descritivos do VI, em substituição à densidade, possibilitou a reclassificação das espécies de duas maneiras distintas: maior destaque das espécies com melhor condição geral das árvores, expressando melhor a adaptação delas à arborização de ruas da cidade (indicada por uma melhor posição no ranking do VI), e depreciação do VI das espécies que apresentaram condição geral das árvores do tipo ruim, a qual expressa uma baixa adaptação às condições da arborização de ruas. Ressalta-se que este método (S4) diferiu significativamente dos demais mediante análise post hoc dos dados, com $p$-valores iguais a $7,9^{-15}, 1,2^{-4}$ e $3,4^{-14}$, respectivamente, em relação a $\mathrm{S} 1, \mathrm{~S} 2$ e S3, além de apresentar os menores valores de correlação por postos com os demais métodos (Tabela 3).

Esta maneira de expressão do VI, por meio do IPE, poderia ajudar no planejamento de ações de manejo voltadas à qualidade da condição apresentada pelas espécies na arborização de ruas, priorizando ações de substituição, seja de árvores adultas que tenham problemas crônicos com pragas e/ou doenças, seja de árvores adultas com qualidade geral depreciada por práticas inadequadas de manejo, seja de mudas ou árvores jovens com qualidade de condição geral depreciada por insatisfatória adaptação ecofisiológica ao meio urbano e/ou devido à baixa qualidade dos indivíduos plantados.

Freilicher (2010) em estudo conduzido para avaliar medidas de performance da arborização de ruas em nove cidades do estado de Massachusetts, Estados Unidos, fez uso do índice de performance da espécie para avaliar a condição geral das árvores de cada espécie em relação à condição geral das árvores dos inventários analisados. Neste trabalho, observou que a espécie com menor performance relativa foi aquela mais comumente observada nos inventários analisados. Os dados obtidos para a cidade de Curitiba demonstraram que Ligustrum lucidum (alfeneiro) assumiu uma tendência semelhante, sendo constatada uma elevada importância indicada pelo VI composto por meio do DAP, mas ao mesmo tempo se destacou como uma das espécies com os menores valores de performance relativa (Tabela 2).

Para as situações S1, S2 e S3, constatou- 
se não haver correlação significativa com a performance das espécies representada pelos valores de IPE (Tabela 4). Isso evidencia que a forma tradicional de se demonstrar a importância das espécies nos inventários da arborização de ruas, pela proporção de indivíduos (SAMPAIO; DE ANGELIS, 2008; ROSSATTO et al., 2008; SILVA et al., 2008; MOURA; SANTOS, 2009; LIMA; SILVA JUNIOR, 2011; SOUZA, 2011; THAIUTSA et al., 2008; SREETHERAN et al., 2011; KURUNERICHITEPO; SHACKLETON; 2011), não expressa satisfatoriamente sua relevância na composição quando o objetivo é considerar a abundância, dimensões e condição das árvores amostradas.

O comportamento das diferentes formas de análise da composição do VI foi detalhado para as principais espécies amostradas (aquelas com maior proporção de indivíduos) estando os resultados representados na Figura 1. As espécies Araucaria angustifolia e Eugenia uniflora não foram inseridas na figura por conta da posição assumida em um dos métodos, o que dificultaria a adequação da escala e da legibilidade da informação.

Para as espécies Tipuana tipu (tipuana), Syagrus romanzoffiana (jerivá), Parapiptadenia rigida (angico), Melia azedarach (cinamomo), Ligustrum lucidum (alfeneiro), Jacaranda mimosifolia (jacarandá-mimoso) e Anadenanthera colubrina (monjoleiro) houve melhoria de posicionamento na ordenação de VI composto a partir do DAP como fator descritivo da dominância (S2) em relação ao "ranqueamento" feito apenas com a frequência relativa (abundância) da espécie (S1).

Para os posicionamentos do VI composto a partir da área de copa como fator descritivo da dominância (Método S3), em relação ao Método S2, foi constatada melhoria de posição apenas para as espécies Tipuana tipu, Poincianella pluviosa (sibipiruna), Jacaranda mimosifolia (jacarandámimoso) e Cassia leptophylla (falso-barbatimão). Por outro lado, a expressão da menor importância (piora no posicionamento) observada para Syagrus romanzoffiana se deve ao fato de que a projeção de copa é proporcionalmente pequena, mesmo para diâmetros do estipe mais avantajados. Quanto à Melia azedarach e Ligustrum lucidum, a expressão da menor importância (correspondente a uma piora no posicionamento) se deve ao fato de que na cidade de Curitiba são espécies que sofrem modificações corriqueiras na área de copa por conta de sucessivas podas realizadas, principalmente de árvores localizadas sob fiação (BOBROWSKI, 2011a).

Quando considerado o VI obtido por meio do índice performance da espécie (Método S4), os piores posicionamentos assumidos em cada espécie (Figura 1) foram observados para Senna macranthera (fedegoso), Poincianella pluviosa (sibipiruna), Ligustrum lucidum (alfeneiro), Lagerstroemia indica (extremosa), Lafoensia pacari (dedaleiro), Hibiscus rosa-sinensis (hibisco), Handroanthus chrysotrichus (ipê-amarelo-miúdo) e Acer negundo (acer). Os melhores posicionamentos assumidos pelas espécies, para este mesmo método de obtenção do VI, foram constatados para Tipuana tipu (tipuana), Parapiptadenia rigida (angico), Melia azedarach (cinamomo), Libidibia ferrea (pauferro), Jacaranda mimosifolia (jacarandá-mimoso), Handroanthus albus (ipê-amarelo-graúdo), Cassia leptophylla (falso-barbatimão) e Anadenanthera colubrina (monjoleiro).

Para as espécies com os piores posicionamentos no Método S4 são destacados os seguintes problemas na arborização (BIONDI; ALTHAUS, 2005): baixa longevidade no meio urbano por intolerância à geada e suscetibilidade a viroses (Senna macranthera); necessidade de podas constantes quando plantada sob rede de transmissão

TABELA 4: Análise de correlação múltipla entre os valores relativos de Indice de Performance da Espécie (IPE) e os diferentes métodos de obtenção da importância das espécies, métodos S1, S2, S3 e $\mathrm{S} 4$, com respectivos valores de significância ( $p$-valor).

TABLE 4: Multiple correlation analysis between relative values of Species Performance Index (IPE) and different methods of obtaining the importance of species, methods S1, S2, S3 e S4, with respective values of significance ( $p$-value).

\begin{tabular}{ccccc}
\hline & $\mathrm{S} 1$ & $\mathrm{~S} 2$ & $\mathrm{~S} 3$ & $\mathrm{~S} 4$ \\
\hline \multirow{2}{*}{ IPE } & 0,02 & 0,04 & 0,02 & 0,18 \\
& $p$-valor $(0,7920)$ & p-valor $(0,6385)$ & p-valor $(0,7863)$ & $p$-valor $(0,0536)$ \\
\hline
\end{tabular}


de energia elétrica (Handroanthus chrysotrichus), possivelmente por seu eixo ortotrópico de crescimento e suscetibilidade a algumas pragas e doenças; intolerância a alguns poluentes atmosféricos, podendo a poda ser um problema para a espécie (Acer negundo) devido ao ataque de fungos em galhos mais velhos. De acordo com Bobrowski (2011a) e Bobrowski e Biondi (2012), Ligustrum lucidum e Lagerstroemia indica são espécies que sofrem, mais intensamente, modificações na copa por conta de podas drásticas executadas, o que deprecia a condição geral das árvores.

A importância da espécie, indicada por sua posição no ranking do VI com dominância calculada pelo DAP ou pela área de copa, representa apenas sua presença na composição da arborização de ruas e não detalha sua real significância e expressividade, principalmente em termos de estabilidade e risco oferecido. Alguns autores relatam a importância da boa condição geral e/ou boa estabilidade estrutural das árvores na arborização de ruas, a fim de reduzir ou evitar danos aos pedestres, veículos e estruturas urbanas (ELLISON, 2005; TERHO, 2009; KONTOGIANNI et al., 2011; BRAZOLIN et al., 2011). Neste sentido, árvores em condições ruins, mas com altos valores de VI, estariam expressando uma falsa importância no contexto de composição da arborização de ruas. Conforme verificado para as espécies acima descritas, o VI composto por meio do IPE em substituição à densidade, ao depreciar o posicionamento das espécies com condições relativamente piores, dá um indicativo mais realista da importância das mesmas na composição da arborização de ruas.

As análises feitas demonstram que a forma tradicionalmente adotada para se expressar a importância das espécies (Método S1) nos inventários da arborização urbana (SAMPAIO; DE ANGELIS, 2008; ROSSATTO et al., 2008; SILVA et al., 2008; THAIUTSA et al., 2008;
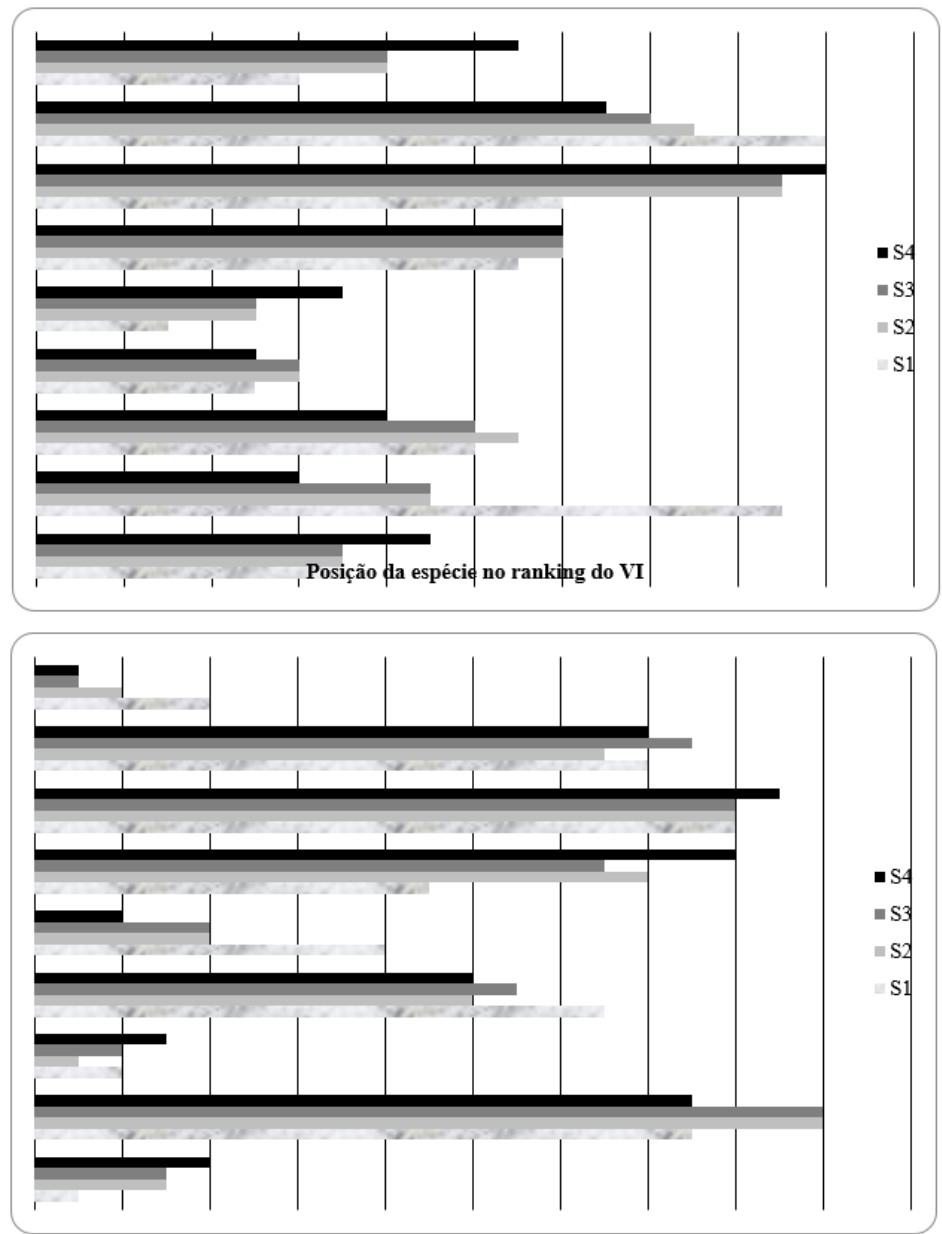

FIGURA 1: Mudanças na posição das espécies nos diferentes métodos de obtenção do valor de importância. FIGURE 1: Changes in position of species in different methods of obtaining the importance value. 
MOURA; SANTOS, 2009; KURUNERICHITEPO; SHACKLETON, 2011; LIMA; SILVA JUNIOR, 2010; SOUZA, 2011; SREETHERAN et al., 2011; KRAMER; KRUPEK, 2012) tendem a depreciar a real importância assumida pelas espécies no contexto de análise e da sua contribuição aos possíveis benefícios gerados. Da mesma forma, se adotada a prática de uso do índice de valor de importância obtido por meio da área basal (Método S2) como fator descritivo da dominância das espécies, muitas delas deixam de assumir relevância, por deixar mascarado o principal meio de origem dos benefícios ambientais e estéticos que é a copa da árvore.

\section{CONCLUSÕES}

$\mathrm{Na}$ arborização de ruas, a classificação da importância das espécies a partir da Área de Copa $\left(\mathrm{m}^{2}\right)$ como descritor do fator dominância do VI e a partir do Índice de Performance da Espécie (IPE) como descritor do fator densidade representaram melhor a contribuição relativa das espécies na composição da comunidade arbórea (abundância, cobertura e condição geral) em relação aos demais métodos analisados, o que pode possibilitar melhores ações de planejamento e gestão do patrimônio arbóreo inserido nas calçadas.

\section{REFERÊNCIAS BIBLIOGRÁFICAS}

ARAUJO, M. M. et al. Análise de agrupamento em remanescente de floresta ombrófila mista. Ciência Florestal, Santa Maria, v. 20, n. 1, p. 1-18, 2010. BIONDI, D.; ALTHAUS, M. Árvores de rua de Curitiba: cultivo e manejo. Curitiba: FUPEF, 2005. BOBROWSKI, R. Estrutura e dinâmica da arborização de ruas de Curitiba, Paraná, no período 1984-2010. 2011. 144 f. Dissertação (Mestrado em Engenharia Florestal) - Setor de Ciências Agrárias, Universidade Federal do Paraná, Curitiba, 2011a.

BOBROWSKI, R. Inventário florestal contínuo e dinâmica da arborização de ruas. In: BIONDI, D.; LIMA NETO, E. M. (Ed.). Pesquisa em arborização de ruas. Curitiba: O Autor, 2011b. p. 109-130.

BOBROWSKI, R.; BIONDI, D. Distribuição e dinâmica da área de copa na arborização de ruas de Curitiba, Paraná, Brasil, no período de 1984-2010. Revista Árvore, Viçosa, v. 36, n. 4, p. 625-635, 2012.
BOBROWSKI, R.; BIONDI, D.; FIGUEIREDO FILHO, A. Dinâmica da distribuição diamétrica na arborização de ruas da cidade de Curitiba, Paraná, Brasil. Scientia Forestalis, Piracicaba, v. 40, n. 94, p. 167-178, 2012.

BRAZOLIN, S. et al. Avaliação do lenho biodeteriorado de árvores de tipuana (Tipuana tipu) em área urbana: análise macroscópica e massa específica aparente. Scientia Forestalis, Piracicaba, v. 9, n. 91, p. 291-299, 2011.

COSTA, M. P. et al. Estrutura e diversidade da comunidade arbórea de uma floresta superomontana, no planalto de Poços de Caldas (MG). Ciência Florestal, Santa Maria, v. 21, n. 4, p. 711-725, 2011. DAVEY RESOURCE GROUP. Calculated Public Tree Values and Benefits for the City of Grand Rapids. Kent: Davey Tree Expert Company, 2010. $65 \mathrm{p}$.

COSTA, M. P. Redwood City, street tree resource analysis. Atascadero: Davey Tree Expert Company, 2012. $60 \mathrm{p}$.

DISALVO, A.; FUCHS, D.; SCHULL, C. Street tree inventory report: Northwest District Neighborhood. Portland: Portland Parks \& Recreation Urban Forestry, 2012. 27 p. Disponível em: $\quad<$ http://www.portlandoregon.gov/parks/ article/419665>. Acesso em: 8 jan. 2013.

FREILICHER, M. E. Evaluating federal urban forestry performance measures in Massachussetts (U.S.A.). 2010. 82 f. Dissertação (Mestrado em Recursos Florestais) - Universidade de Massachusetts, Amherst, 2010.

GALVIN, M. F. A report on Hyattsville's street tree. Annapolis: Maryland Department of Natural Resources-Forest Service, 2007. 13 p. Disponível em: <http://www.dnr.state.md.us/irc/ docs/00013583.pdf > . Acesso em: 8 jan. 2013.

HASENAUER, H. Dimensional relationships of open-grown trees in Austria. Forest Ecology and Management, Amsterdam, v. 96, n. 3, p. 197-206, 1997.

KRAMER, J. A.; KRUPEK, R. A. Caracterização florística e ecológica da arborização de praças públicas do município de Guarapuava, PR. Revista Árvore, Viçosa, MG, v. 36, n. 4, p. 647-658, 2012. KURUNERI-CHITEPO, C.; SHACKLETON, C. $M$. The distribution, abundance and composition of street trees in selected towns of the Eastern Cape, South Africa. Urban Forestry \& Urban Greening, Amsterdam, v. 10, n. 3, p. 247-254, 2011.

LIMA, R. M. C.; SILVA JUNIOR, M. C. Inventário da arborização urbana implantada na década de 60 
no plano piloto, Brasília, DF. Revista da Sociedade Brasileira de Arborização Urbana, Piracicaba, v. 5, n. 4, p. 110-127, 2010.

$\mathrm{MACO}, \mathrm{S}$. E. A practical approach to assessing structure, function, and value of street tree populations in small communities. 2003. $218 \mathrm{f}$. Dissertação (Mestrado em Conservação Florestal) Departamento de Silvicultura e Proteção do Fogo, Universidade da Califórnia, Davis, 2003.

MARTINI, A.; BIONDI, D.; BATISTA, A. C. Variação diária e estacional do microclima urbano em ruas arborizadas de Curitiba-PR. Floresta e Ambiente, Seropédica, v. 20, n. 4, p. 460-469, 2013. MARTINI, A. et al. A periodicidade diária do índice de conforto térmico na arborização de ruas de Curitiba-PR. Floresta e Ambiente, Seropédica, v. 9, n. 5, p. 1-9, 2013.

MARTINS, A. K. E. et al. Relações sologeoambiente em áreas de ocorrências de Ipucas na planície do Médio Araguaia - Estado de Tocantins. Revista Árvore, Viçosa, v. 30, n. 2, p. 297-310, 2006.

MCLEOD, A. I. Kendall rank correlation and Mann-Kendall trend test. [s. 1.]: R. package, 2011. Disponível em: <http://CRAN.R-project.org/ package=Kendall $>$. Acesso em: 20 jan. 2015.

MCPHERSON, E. G.; SIMPSON, J. R. A comparison of municipal forest benefits and costs in Modesto and Santa Monica, California, USA. Urban Forestry \& Urban Greening, Amsterdam, v. 1, n. 2, p. 61-74, 2002.

MCPHERSON, E. G. et al. Benefit-cost analysis of Fort Collin's Municipal Forest. Davis: Center for Urban Forest Research/ USDA Forest Service, $2003.39 \mathrm{p}$.

MCPHERSON, E. G. et al. Municipal forest benefits and costs in five US cities. Journal of Forestry, Bethesda, v. 103, n. 8, p. 411-416, 2005.

MORO, M. F.; MARTINS, F. R. Métodos de levantamento do componente arbóreo-arbustivo. In: FELFILI, J. M. et al. Fitossociologia no Brasil: métodos e estudos de casos. Viçosa: Editora UFV, 2011. p. 174-212.

MOURA, T. A.; SANTOS, V. L. L. V. Levantamento quali-quantitativo de espécies arbóreas e arbustivas na arborização viária urbana dos bairros centro e centro norte, Várzea Grande, Mato Grosso, Brasil. Revista da Sociedade Brasileira de Arborização Urbana, Piracicaba, v. 1, n. 1, p. 97-117, 2009.

MUTHULINGAM, U.; THANGAVEL, S. Density, diversity and richness of woody plants in urban green spaces: A case study in Chennai metropolitan city. Urban Forestry \& Urban Greening, Amsterdam, v. 11, n. 4, p. 450-459, 2012.

NAGENDRA, H.; GOPAL, D. Street trees in Bangalore: Density, diversity, composition and distribution. Urban Forestry \& Urban Greening, Amsterdam, v. 9, n. 2, p. 129-137, 2010.

NOWAK, D. J. et al. A ground based method for assessing urban forest structure and ecosystem services. Arboriculture \& Urban forestry, Champaign, v. 34, n. 6, p. 347-358, 2008.

PEPER, P. J. et al. City of Bismarck, North Dakota Street Tree Resource Analysis. Davis: Center for Urban Forest Research/ USDA Forest Service, 2004. $64 \mathrm{p}$.

POHLERT, T. The Pairwise Multiple Comparison of Mean Ranks Package (PMCMR). [s. 1.]: R. package, 2014. Disponível em: <http://CRAN.Rproject.org $/$ package $=$ PMCMR $>$. Acesso em: 20 jan. 2015.

RAUPP, M. J.; CUMMING, A. B.; RAUPP, E. C. Street tree diversity in eastern north America and its potential for tree loss to exotic borers. Arboriculture \& Urban Forestry, Champaign, v. 32, n. 6, p. 297-304, 2006.

$R$ CORE TEAM R. A language and environment for statistical computing. Viena: R Foundation for Statistical Computing, 2014. Disponível em: $<\mathrm{http} / / /$ www.R-project.org>. Acesso em: 20 jan. 2015.

RICHARDS, N. A. Reasonable guidelines for street tree diversity. Journal of Arboriculture, Champaign, v. 19, n. 6, p. 344-350, 1993.

ROMANI, G. N. Análise florística fitossociológica e qualitativa da arborização na Praça XV de Novembro em Ribeirão Preto, SP. 2011. 61 f. Dissertação (Mestrado em Agronomia - Produção Vegetal) - Faculdade de Ciências Agrárias e Veterinárias, Universidade Estadual Paulista Julio de Mesquita Filho, Jaboticabal, 2011.

ROSSATTO, D. R.; TSUBOY, M. S. F.; FREI, F. Arborização urbana na cidade de Assis-SP: uma abordagem quantitativa. Revista da Sociedade Brasileira de Arborização Urbana, Piracicaba, v. 3, n. 3, p. 1-16, 2008.

SAMPAIO, A. C. F.; DE ANGELIS, B. L. D. Inventário e análise da arborização de vias públicas de Maringá-PR. Revista da Sociedade Brasileira de Arborização Urbana, Piracicaba, v. 3, n. 1, p. 37-57, 2008.

SARTORI, R. A.; BALDERI, A. P. Inventário da arborização urbana do município de Socorro-SP e proposta de um índice de danos à infraestrutura 
das cidades. Revista da Sociedade Brasileira de Arborização Urbana, Piracicaba, v. 6, n. 4, p. 68-98, 2011.

SCHALLENBERGER. L. S.; MACHADO, G. O. Inventário da arborização na região central do município de Mangueirinha-PR. Revista da Sociedade Brasileira de Arborização Urbana, Piracicaba, v. 8, n. 1, p. 54-64, 2014.

SEMENZATO, P.; CATTANEO, D.; DAINESE, M. Growth prediction for five tree species in an Italian urban forest. Urban Forestry \& Urban Greening, Amsterdam, v. 10, n. 3, p. 169-176, 2011.

SIEGEL, S.; CASTELLAN, N. J. Estatística nãoparamétrica para ciências do comportamento. Porto Alegre: Artmed, 2006.

SILVA, A. C. et al. Relações florísticas e fitossociológicas de uma floresta ombrófila mista montana secundária em Lages, Santa Catarina. Ciência Florestal, Santa Maria, v. 22, n. 1, p. 193-206, 2012.

SILVA, L. M. et al. Inventário da arborização em duas vias de Mariápolis/PR. Revista da Sociedade Brasileira de Arborização Urbana, Piracicaba, v. 3, n. 1, p. 36-53, 2008.

SILVA, M. D. M.; SILVEIRA, R. P.; TEIXEIRA, M. I. J. G. Avaliação da arborização de vias públicas de uma área da região oeste da cidade de Franca/SP. Revista da Sociedade Brasileira de Arborização Urbana, Piracicaba, v. 3, n. 1, p. 19-35, 2008.
SOARES, A. L. et al. Benefits and costs of street trees in Lisbon, Portugal. Urban Forestry \& Urban Greening, Amsterdam, v. 10, n. 2, p. 69-78, 2011.

SOUSA, L. M.; FIGUEIRÊDO, M. F.; BRAGA, P. E. T. Levantamento quali-quantitativo da arborização urbana do distrito de Rafael Arruda, Sobral, CE. Revista da Sociedade Brasileira de Arborização Urbana, Piracicaba, v. 8, n. 3, p. 118-129, 2013.

SOUZA, W. Caracterização da cobertura arbórea dos Parques Urbanos da cidade de Recife - PE. 2011. 101 f. Tese (Doutorado em Ciências Florestais) - Universidade Federal Rural de Pernambuco, Departamento de Ciência Florestal, Recife, 2011.

SREETHERAN, M.; ADNAN, M.; KHAIRIL AZUAR, A. K. Street Tree Inventory and Tree Risk Assessment of Selected Major Roads in Kuala Lumpur, Malaysia. Arboriculture \& Urban Forestry, Champaign, v. 37, n. 5, p. 226-235, 2011.

TERHO, M. An assessment of decay among urban Tilia, Betula, and Acer trees felled as hazardous. Urban Forestry \& Urban Greening, Amsterdam, v. 8, n. 2, p. 77-85, 2009.

THAIUTSA, B. et al. Urban green space, street tree and heritage large tree assessment in Bangkok, Thailand. Urban Forestry \& Urban Greening, Amsterdam, v. 7, n. 3, p. 219-229, 2008. 